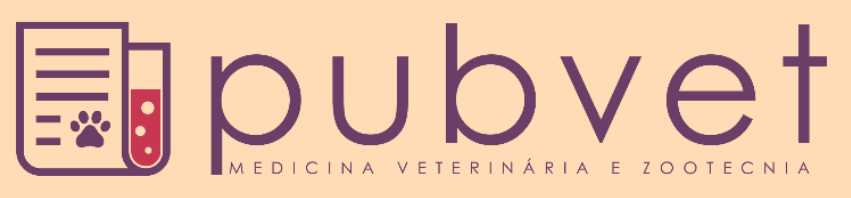

ISSN 1982-1263

https://doi.org/10.31533/pubvet.v13n4a307.1-8

\title{
Leishmaniose visceral canina: Relato de caso
}

\author{
Thaís Carneiro Abbiati ${ }^{1}$, Dilma Mendes de Freitas ${ }^{2 *}$, Laiane Cintra Alves ${ }^{1}$, Bruna Gonzaga de \\ Freitas $^{1 \bullet}$, Rodrigo Supranzetti de Rezende ${ }^{3}$, Stella Góis Barbosa ${ }^{4}{ }^{\ominus}$, Ana Luiza Teixeira

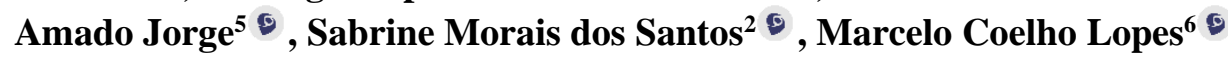 \\ ${ }^{I}$ Médica Veterinária graduada pela Universidade de Uberaba. Uberaba - MG Brasil. \\ ${ }^{2}$ Residente do Programa de Aprimoramento em Patologia Animal do Hospital Veterinário de Uberaba. Uberaba-MG Brasil. \\ ${ }^{3}$ Docente do curso de Medicina Veterinária na Universidade de Uberaba. Uberaba-MG Brasil. \\ ${ }^{4}$ Médica Veterinária e Proprietária da Clínica AmisVet de Uberaba. Uberaba - MG Brasil. \\ ${ }^{5}$ Médica Veterinária graduada pela Universidade Presidente Antônio Carlos. Uberlândia-MG Brasil. \\ ${ }^{6}$ Mestrando em Patologia Animal, Universidade Federal de Minas Gerais. Belo Horizonte - MG Brasil. \\ *Autor para correspondência: E-mail: dilmamendes3m@yahoo.com.br
}

Resumo. A Leishmaniose Visceral Canina (LVC) é causada por um protozoário do gênero Leishmania spp., é uma grave doença que atinge várias espécies, inclusive o cão que é o maior reservatório do meio urbano e o homem. É transmitida pela picada de flebotomíneos onde a forma promastigota ocorre no vetor e a forma amastigota ocorre no hospedeiro vertebrado. Os sinais clínicos se manifestam de acordo com o grau de infestação e imunidade do hospedeiro. O diagnóstico envolve testes rápidos, testes parasitológicos e sorológicos. O tratamento tem custo elevado e são necessários cuidados constantes devido a possibilidade de retorno dos sinais clínicos. A melhor forma de se combater a doença é através de medidas que visam o controle do vetor e proteção dos animais. O objetivo deste trabalho é relatar um caso de um cão da raça Border Collie de 3 anos de idade, com histórico de apresentar epistaxe recorrente, alopecia periocular, lesões em narinas, seborreia seca nas orelhas e região cervico-torácica, proveniente do município de Uberaba, atendida na Clínica AmisVet, abordando a clínica do animal, o diagnóstico e o tratamento. O exame ELISA confirmou o diagnóstico de Leishmaniose Visceral Canina. O tratamento foi realizado com miltefosina. $\mathrm{O}$ animal apresentou excelente recuperação durante o período de tratamento. Conclui-se que são importantes a realização de exames complementares, pois nem todos os animais são sintomáticos e o tratamento recomendado é medicamentoso.

Palavras chave: Lutzomya longipalpis, cão, miltefosina, dermatite

\section{Visceral canine leishmaniasis: Case report}

Abstract. Visceral Canine Leishmaniasis (LVC) is caused by a protozoan of the genus Leishmania spp., Is a serious disease that affects several species, including the dog that is the largest reservoir of the urban environment and man. It is transmitted by the sting of sandflies where the promastigote form occurs in the vector and the amastigote form occurs in the vertebrate host. Clinical signs manifest themselves according to the degree of infestation and immunity of the host. Diagnosis involves rapid tests, parasitological and serological tests. Treatment is expensive and constant care is needed because of the possibility of clinical signs returning. The best way to combat the disease is through measures aimed at vector control and animal protection. The objective of this work is to report a case of a 3-year-old Border Collie dog with a history of recurrent epistaxis, periocular alopecia, lesions in the nostrils, dry seborrhea in the ears and cervico-thoracic region, coming from the municipality of Uberaba, attended at the AmisVet Clinic, addressing the animal's clinic, diagnosis and treatment. The ELISA confirmed the diagnosis 
of Visceral Canine Leishmaniasis. The treatment was performed with miltefosine. The animal presented excellent recovery during the treatment period. It is concluded that complementary tests are important, since not all animals are symptomatic and the recommended treatment is medicated.

Keywords: Lutzomya longipalpis, dog, miltefosine, dermatitis

\section{Leishmaniasis visceral canina: Reporte de caso}

Resumen. La Leishmaniasis Visceral Canina (LVC) es causada por un protozoario del género Leishmania spp., Es una grave enfermedad que afecta a varias especies, incluso el perro que es el mayor reservorio del medio urbano y el hombre. Es transmitida por la picadura de flebotomíneos donde la forma promastita ocurre en el vector y la forma amastigotes ocurre en el huésped vertebrado. Los signos clínicos se manifiestan de acuerdo con el grado de infestación e inmunidad del huésped. El diagnóstico involucra pruebas rápidas, pruebas parasitológicas y serológicas. El tratamiento tiene un costo elevado y se requiere atención constante debido a la posibilidad de retorno de los signos clínicos. La mejor forma de combatir la enfermedad es a través de medidas que apuntan al control del vector y protección de los animales. El objetivo de este trabajo es relatar un caso de un perro de la raza Border Collie, con edad de 3 años, con historial de presentar epistaxis recurrente, alopecia periocular, lesiones en narinas, seborrea seca en las orejas y región cervico-torácica, proveniente del municipio de Uberaba, atendida en la Clínica AmisVet, abordando la clínica del animal, el diagnóstico y el tratamiento. El examen ELISA confirmó el diagnóstico de Leishmaniosis Visceral Canina. El tratamiento se realizó con miltefosina. El animal presentó una excelente recuperación durante el período de tratamiento. Se concluye que son importantes la realización de exámenes complementarios, pues no todos los animales son sintomáticos y el tratamiento recomendado es medicamentoso.

Palabras clave: Lutzomya longipalpis, perro, miltefosina, dermatites

\section{Introdução}

A leishmaniose visceral canina (LVC) é uma antropozoonose causada pelo protozoário do gênero Leishmania spp. e transmitida pela picada do flebotomíneo (Lutzomyia longipalpis), onde o cão é o principal reservatório (Silva, 2007). A Leishmania infantum é considerado o principal agente etiológico da leishmaniose canina (Solano-Gallego et al., 2011). O parasita tem hábitos noturnos e seus ovos são depositados em ambientes úmidos e ricos em matéria orgânica (Ciaramella \& Corona, 2003; Madeira et al., 2003). O vetor quando se alimenta de um animal infectado ingere o protozoário na forma amastigota e transforma-se em promastigota, continuando o ciclo do parasita (Abrantes \& Silveira, 2009).

O animal infectado pode se encontrar clinicamente saudável por um longo período, mas permanece como reservatório da doença e com a capacidade de infectar o vetor e continuar com a disseminação do ciclo (Gontijo \& Melo, 2004; Mancianti et al., 1988). Os sinais clínicos mais observados incluem lesões cutâneas tais como alopecia, descamação, hiperqueratose nasal, úlceras e hiperpigmentação, além de anorexia, onicogrifose e alterações oftálmicas (Koutinas \& Koutinas, 2014).

O diagnóstico é realizado por exames RIFI, ELISA, PCR, teste rápido e observação direta da forma amastigota do protozoário pela análise de esfregaço ( Mohapatra et al., 2014; Dantas-Torres et al., 2017).

Com o tratamento, ocorre melhora clínica e diminuição da carga parasitária no animal, mas não o torna livre de ser um reservatório e não impede que os sinais clínicos não voltem (Baneth \& Shaw, 2002).

A prevenção requer a conscientização das pessoas sobre a gravidade da doença para que se possam tomar medidas no controle do vetor para proteção dos animais nessas áreas, com uso de coleiras e pouron com inseticidas e repelentes, vacina e pulverização do ambiente (Werneck et al., 2002).

O objetivo deste trabalho é relatar o caso de um cão de três anos de idade com leishmaniose visceral, atendido na Clínica AmisVet, abordando a clínica do animal, o diagnóstico e o tratamento. 


\section{Relato de caso}

Foi atendido na Clínica AmisVet, um cão da raça Border Collie, de três anos de idade, com peso corporal de $17 \mathrm{~kg}$, procedente do município de Uberaba. A queixa principal era que esse animal apresentava lesões com presença de crostas, eritema e hiperqueratose. As lesões se distribuíam na região mentoniana, perilabial, narina e ponta de orelha. A pele estava com aspecto descamativo principalmente em axilas, virilha, membros anteriores e posteriores, queda de pêlos ao redor do olho e cauda. O tutor relatou também dificuldade respiratória com constipação e epistaxe unilateral. $O$ animal foi diagnosticado por outro profissional com artrite e faz uso contínuo de regenerador osteoarticular. Havia queixa de dor e dificuldade de locomoção em membros posteriores.

Este paciente já havia passado por consulta com outros médicos veterinários, com suposto diagnóstico de hemoparasitose e trombocitopenia imunomediada, sendo realizados tratamentos com antibioticoterapia, antianêmicos, corticoide e imunossupressor, sem resultado satisfatório. Informou que o cão apresentava normorexia, normodipsia, normoquesia e normúria. Foi mencionado que o animal toma banho em casa. A alimentação consistia de ração e comida caseira. Além disso, confirmou que o animal tem acesso a rua e contato com terra, vivendo em um ambiente urbano e convivendo com outro coabitante canino assintomático. Refere que a derverminação e a vacinação estão atualizados e encontrou carrapato no animal recentemente.

No exame clínico geral, o animal apresentava-se alerta, ativo, mucosas hipocoradas, hidratação normal, TPC de 2 segundos, linfonodos submandibulares, pré-escapulares e poplíteo estavam reativos, frequência cardíaca de $120 \mathrm{bpm}$, frequência respiratória de $28 \mathrm{mpm}$ e temperatura retal de $38,5^{\circ} \mathrm{C}$. Não foram identificadas alterações a palpação abdominal.

Durante o exame físico foram observados dificuldade de locomoção em membros pélvicos, dificuldade respiratória, epistaxe unilateral, alopecia periocular e em ponta de cauda, hiperqueratose nasal, lesões em região mentoniana e perilabial, dermatite descamativa em ponta de orelha, região cervico-torácica, inguinal, membros anteriores e posteriores (Figura 1), suspeitando-se de leishmaniose visceral canina e hemoparasitose.

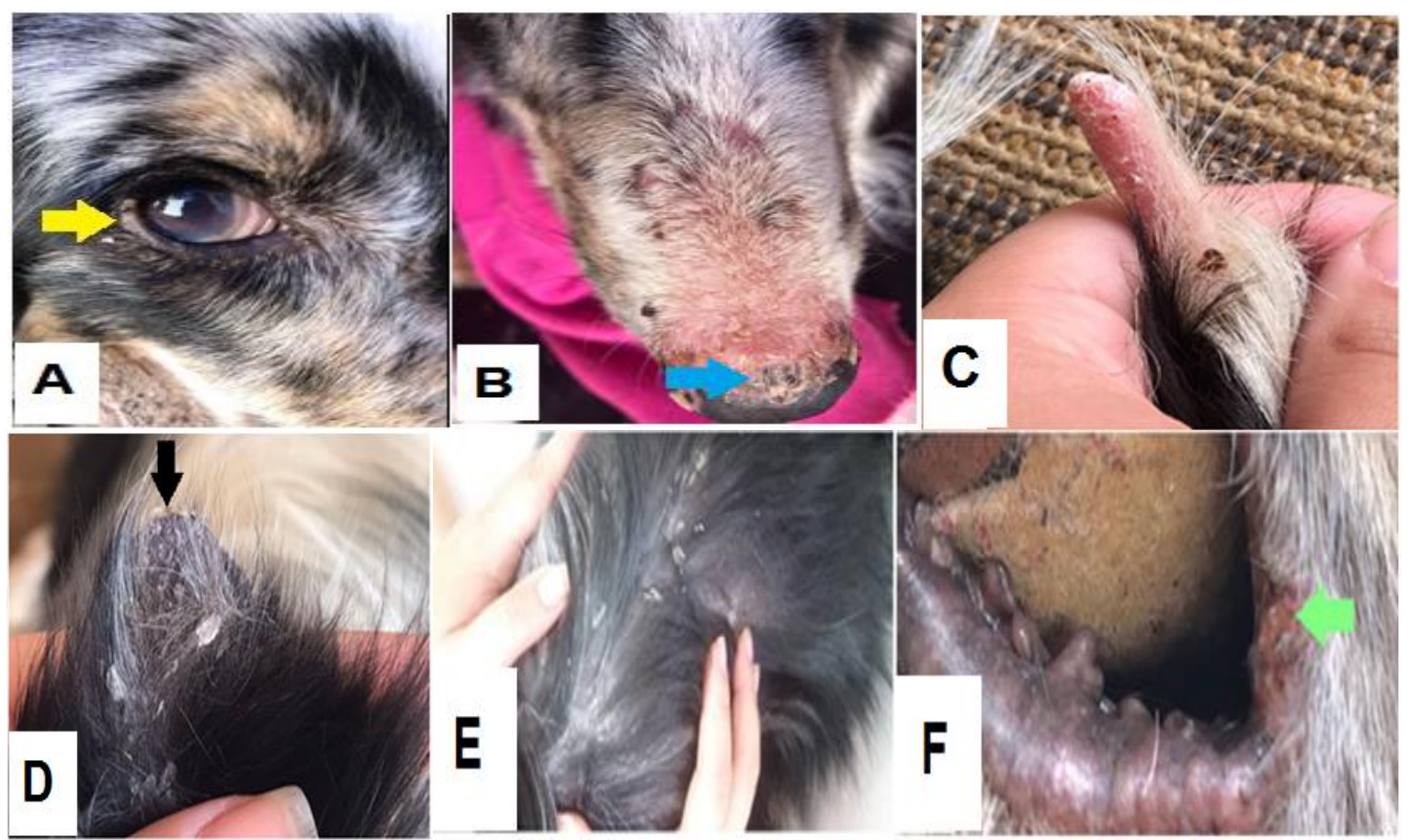

Figura 1. Imagem de um cão da raça Border Collie, de 3 anos de idade, atendido no dia 01 de agosto de 2018, na Clínica AmisVet em Uberaba-MG. A) Alopecia periocular (seta amarela). B) Hiperqueratose nasal (seta azul). C) Alopecia em ponta de cauda. D) Dermatite descamativa em ponta de orelha (seta preta). E) Dermatite descamativa em região cervico-torácica. F) Lesão em região perilabial (seta verde). 
Para tratamento tópico, foi prescrito shampoo a base de clorexidina e miconazol para banhos a cada 5 dias totalizando quatro banhos, hidratante a base de óleo de macadâmia, ceramidas e silicone, além de antibiótico rifamicina spray para borrifar nas lesões cutâneas.

Para uso oral, foi prescrito imunoestimulante a base de timomodulina ( $1 \mathrm{mg} / \mathrm{kg}$, BID, por 20 dias), dipirona ( $25 \mathrm{mg} / \mathrm{kg}$, TID, por 3 dias), ácido tranexâmico ( $15 \mathrm{mg} / \mathrm{kg}$, BID, por cinco dias) e cloridrato de tramadol (3mg/kg, TID, por 10 dias).

Foi solicitado hemograma completo, bioquímico (albumina, ALT, creatinina e ureia), raspado de pele para pesquisa de fungos e ácaros, cultura de fungos, RIFI para Erlichia e Babesia, além de exame ELISA para leishmaniose. No hemograma, observou-se anemia normocitica normocrômica, leucopenia, bastonetes aumentados e trombocitopenia.

No hemograma, observou-se anemia normocitica normocrômica, leucopenia, bastonetes aumentados e trombocitopenia (Tabela 1).

Tabela 1. Perfil hematológico de um cão, da raça Border Collie, de 3 anos de idade, atendido na Clínica AmisVet em UberabaMG, realizado no dia 08 de agosto de 2018, com histórico de lesões cutâneas e diagnóstico final de leishmaniose visceral canina

\begin{tabular}{lcc}
\hline Hemograma & Resultado & Referência \\
\hline Eritrograma & & $5,5-8,5$ \\
Hemácias & $3,60 \mathrm{milhões} / \mathrm{mm}^{3}$ & $12-18$ \\
Hemoglobina & $7,8 \mathrm{~g} / \mathrm{dl}$ & $37-55$ \\
Hematócrito & $23,8 \%$ & $60-77$ \\
VCM & $66,11 \mathrm{fl}$ & $31-37$ \\
CHCM & $32,77 \%$ & $19,0-24,5$ \\
HCM & $21,66 \mathrm{pg}$ & $200.000-600.000$ \\
Plaquetas & $125.000 / \mathrm{mm}^{3}$ & \\
\hline Leucograma & & $6.000-18.000$ \\
\hline Leucócitos & $4.700 / \mathrm{mm}^{3}$ & $60-80$ \\
Neutrófilos & $3.666 / \mathrm{mm}^{3}(78 \%)$ & $00-05$ \\
Bastonetes & $517 / \mathrm{mm}^{3}(11 \%)$ & $60-70$ \\
Segmentados & $3.149 / \mathrm{mm}^{3}(67 \%)$ & $12-30$ \\
Linfócitos & $940 / \mathrm{mm}^{3}(20 \%)$ & $03-10$ \\
Monócitos & $94 / \mathrm{mm}^{3}(2 \%)$ & \\
\hline
\end{tabular}

Fonte: Laboratório Bio Med Uberaba

$\mathrm{Na}$ análise bioquímica, observou-se hipoalbuminemia, creatinina diminuída e demais parâmetros dentro dos valores de referência (Tabela 2).

Tabela 2. Perfil bioquímico de um cão, da raça Border Collie, de 3 anos de idade, atendido na Clínica AmisVet em Uberaba-MG, realizado no dia 10 de agosto de 2018, com histórico de lesões cutâneas e diagnóstico final de leishmaniose visceral canina.

\begin{tabular}{lcc}
\hline Bioquímica & Resultado & Referência \\
\hline Albumina & $0,9 \mathrm{~g} / \mathrm{dL}$ & $2,3-3,8$ \\
ALT & $20 \mathrm{U} / \mathrm{L}$ & $10-88$ \\
Creatinina & $0,8 \mathrm{mg} / \mathrm{dL}$ & $1,0-2,0$ \\
Uréia & $15 \mathrm{mg} / \mathrm{dL}$ & $15,0-40,0$ \\
\hline
\end{tabular}

Fonte: Laboratório Villa Real Pet Shop.

No raspado de pele, o resultado foi positivo para pesquisa de fungos com presença de algumas hifas e resultado negativo para pesquisa de ácaros através de clarificação com hidróxido de potássio. Na cultura de fungos, o resultado foi positivo para Candida spp. pelo método Sabouraud/Mycosel. No perfil para hemoparasitas, o resultado foi negativo para Mycoplasma haemocanis utilizando-se o método Percoll, e o resultado foi não- reagente para Babesia (IgG e IgM) com RIFI e o resultado foi reagente (IgG) e não- reagente (IgM) para Erliquia, com RIFI. No exame ELISA, o resultado foi reagente (IgG) para Leishmania spp (Tabela 3). 
Tabela 3. Exame sorológico de ELISA para pesquisa de Leishmania spp. de um cão, da raça Border Collie, de 3 anos de idade, atendido na Clínica AmisVet em Uberaba-MG, realizado no dia 08 de agosto de 2018, com histórico de lesões cutâneas e diagnóstico final de leishmaniose visceral canina.

\begin{tabular}{lcc}
\hline Leishmaniose & Método & Resultado \\
\hline Leishmania spp. (IgG) & ELISA & Reagente \\
\hline Fonte: TECSA Laborários
\end{tabular}

Fonte: TECSA Laboratórios.

Foi prescrito para uso oral, protetor gástrico omeprazol (1mg/kg, SID, por 28 dias), antibiótico doxiciclina (1 comprimido/20kg, BID, por 28 dias), suplemento vitamínico á base de ácido fólico, vitamina B12 e ferro (1 tablete/animal, SID, por 30 dias), S-Adenosil-Metionina (20 mg/kg, SID, por 30 dias) e suplemento a base de extrato de cardo mariano e associações ( 1 comprimido/10kg, SID, por 30 dias). Para tratamento da leishmaniose foi prescrito miltefosina $2 \%(1 \mathrm{~mL} / 10 \mathrm{~kg}$, SID, por 28 dias), anti-emético ondansetrona ( $1 \mathrm{mg} / \mathrm{kg}$, BID, por 2 dias) para uso somente em caso de vômito e foi recomendado o uso de coleira repelente. O proprietário relatou que o animal estava bem e enviou foto do cão em sua residência (Figura 2).

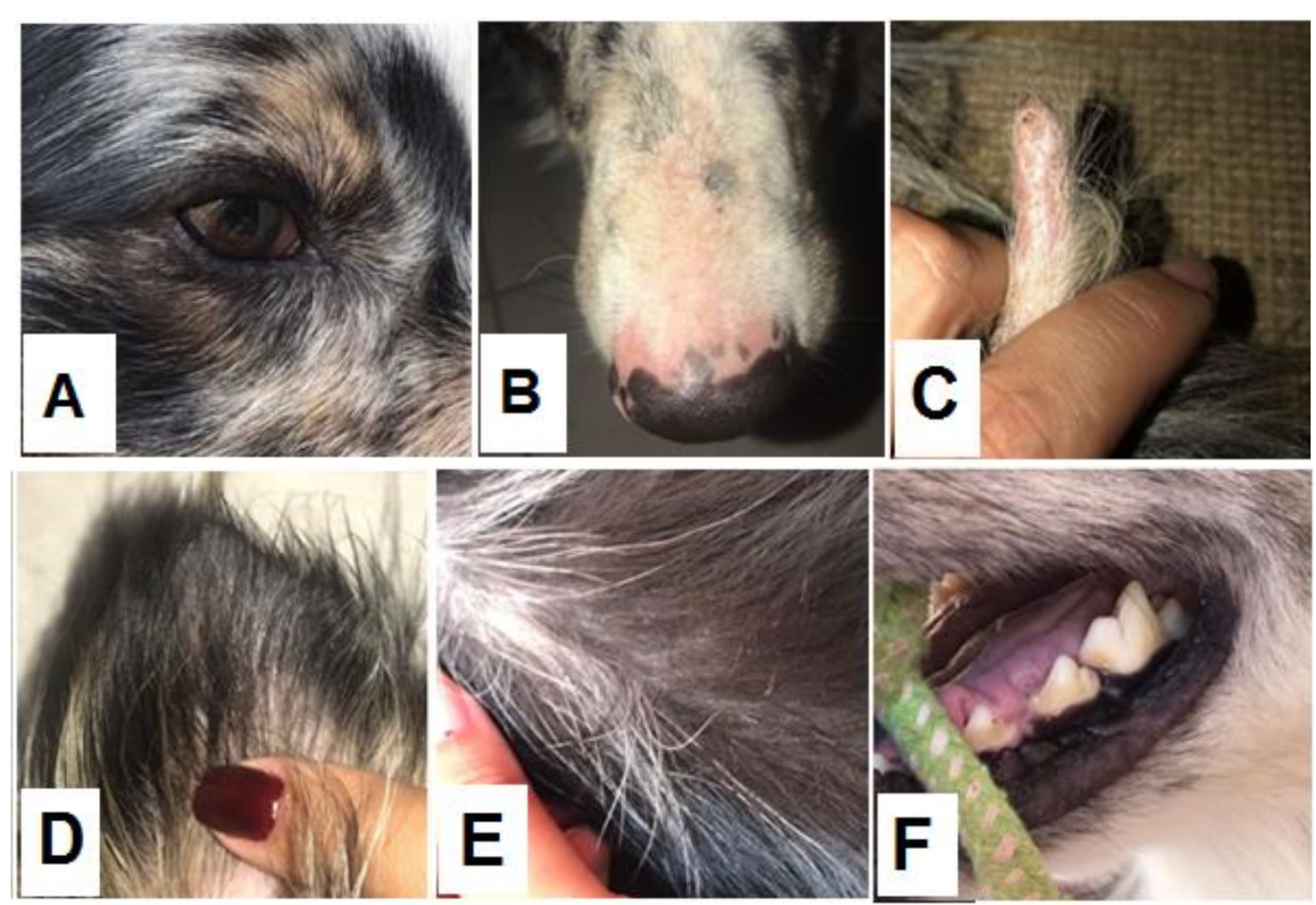

Figura 2. Animal com 15 dias após o término do tratamento, em sua residência. A) Ausência de alopecia periocular. B) Eliminação das lesões em narinas. C) $\mathrm{Na}$ ponta da cauda ainda não houve crescimento total dos pêlos. D) Desaparecimento da dermatite descamativa em ponta de orelha. E) Ausência de dermatite descamativa em região cérvico-torácica. F) Inexistência de lesão em região perilabial.

\section{Discussão}

$\mathrm{O}$ animal do presente relato era proveniente de zona urbana, porém tinha acesso à rua e provavelmente a local com maior risco de infecção. A transmissão ocorre por meio da picada do flebotomíneo que é atraído principalmente por ambientes onde se encontra matéria orgânica (Werneck et al., 2002), portanto essa pode ter sido a forma de infecção. Além disso, há relatos de outros prováveis vetores da doença como pulgas e carrapatos, mas são necessários mais estudos que comprovem como possíveis vetores da doença (Paz et al., 2010).

O paciente deste relato apresentou sintomatologia clínica com lesões cutâneas como alopecia periocular, hiperqueratose nasal, lesão perilabial e em narina, dermatite descamativa em ponta de orelha e região cervico-torácica, além de anemia, hipoalbuminemia, febre, linfadenoapatia e epistaxe unilateral, e foi diagnosticado com leishmaniose visceral canina. Os sinais clínicos descritos condizem com aqueles descridos por Solano-Gallego et al. (2011). 
O animal já tinha passado por consulta com outros profissionais e recebeu o diagnóstico presuntivo de hemoparasitose e trombocitopenia imunomediada baseado apenas no exame de hemograma, e foi realizado o teste ELISA a pedido do tutor que suspeitava de leishmaniose e o resultado foi não reagente. $\mathrm{Na}$ ocasião, foi repetido o teste ELISA, que demonstrou resultado reagente (IgG) para Leishmania spp. e realizado exame de hemograma que demonstrou anemia e trombocitopenia. Sendo assim, foi solicitado sorologia neste relato de caso para pesquisa de hemoparasitas que demonstrou resultado reagente para Erliquia, pois o animal apresentava carrapatos e também em razão de outras alterações como anemia, epistaxe unilateral e trombocitopenia, sendo que esses achados são semelhantes à outras doenças como leishmaniose, o que torna o diagnóstico complexo devido a não existir um teste $100 \%$ sensível e específico (Gontijo \& Melo, 2004). Entretanto, o teste ELISA pode apresentar reação cruzada devido a infecções concomitantes (Ferreira et al., 2007), como aconteceu neste trabalho.

No animal em estudo, não foi realizado o teste rápido e o diagnóstico de leishmaniose foi confirmado por meio do ensaio imuno enzimático (ELISA). Segundo Pinto et al. (2016), a estratégia do programa de controle da leishmaniose visceral canina do Ministério da Saúde inclui o uso do teste rápido associado ao ELISA aumentando a sensibilidade em animais que são positivos. O teste rápido Dual Path Plataform (DPP®) passou a substituir o RIFI como teste de triagem, o PCR identifica o DNA do parasita e no exame parasitológico se faz a observação direta da forma amastigota do protozoário(Benites et al., 2011).

A bioquímica renal (creatinina) deste animal se encontrava abaixo dos valores de referência, porém, não foi realizado exame de urina. A urinálise e o exame de imagem são importantes para identificação de alterações estruturais renais, assim como as enzimas urinárias, que indicam lesão renal (Finco, 1971; Kaneko et al., 2008; Lanis et al., 2008). Os animais com leishmaniose podem desenvolver glomerulonefrite em razão de deposição de imunocomplexos no rim, o que não foi observado no paciente deste relato (Nelson \& Couto, 2015).

$\mathrm{O}$ animal apresentou dificuldade de locomoção e dor nas articulações em membros posteriores durante a palpação e foi prescrito tramadol $(3 \mathrm{mg} / \mathrm{kg}$, TID, por 10 dias). $\mathrm{O}$ animal já havia sido diagnosticado por outro profissional com artrite e após início do tratamento com miltefosina, o tutor relatou o desaparecimento da dor e da dificuldade de locomoção. Os cães com leishmaniose associada a artrite podem exibir severidade variável de claudicação e são descritas as formas erosivas e nãoerosivas (Agut et al., 2003; Blavier et al., 2001). De acordo com Agut et al. (2003), os diferentes padrões radiográficos estão relacionados ao estágio da leishmaniose e/ou resposta imune do animal, sendo que a artrite não erosiva se apresenta nos estágios agudos e subagudos e a erosiva, no estágio crônico. Há uma discordância entre os autores em relação de como acontece a poliartrite desencadeada por Leishmania spp., onde alguns acreditam que ocorra devido a uma resposta inflamatória granulomatosa (McConkey et al., 2002) e outros que seja em razão da deposição de imunocomplexos na articulação (Cucinotta et al., 1991; Ferrer, 1992).

Baseado na intensidade dos sinais clínicos e alterações laboratoriais apresentados pelo animal do presente estudo, a leishmaniose foi classificada como sendo do estágio II. O tratamento foi iniciado com administração de miltefosina ( $2 \mathrm{mg} / \mathrm{kg}$, SID, por 28 dias) e foi prescrito anti-emético ondansetrona (1 $\mathrm{mg} / \mathrm{kg}$, BID) em caso de vômitos. A miltefosina reduz a carga parasitária diminuindo o potencial de infecção, porém o animal continua sendo um reservatório da doença e isso não impede que os sinais clínicos retornem após o tratamento (Baneth \& Shaw, 2002). A miltefosina causa principalmente desordens gastrintestinais como vômito e diarreia (Fischer et al., 2001).

Durante a terapêutica do paciente deste relato, não foi prescrito alopurinol. $\mathrm{O}$ alopurinol é um medicamento para tratamento da leishmaniose visceral humana e, portanto, segundo a legislação vigente com portaria interministerial $\mathrm{n}^{\circ} 1.426$, de $11 / 07 / 08$, não pode ser prescrito para tratamento veterinário. Foi publicado um estudo que demonstrou os efeitos que a terapia com o alopurinol pode causar no sistema urinário, tais como xantinúria, mineralização renal e urolitíase em paciente canino (Torres et al., 2016).

Além do mais, não foi prescrito nenhum imunomodulador durante o tratamento da leishmaniose no animal deste relato, apesar de ser indicado pela literatura. $\mathrm{O}$ tratamento com a domperidona contribui para a modulação da resposta imune inata, melhorando assim os mecanismos de defesa do animal com 
ativação de células fagocíticas e potencializa a destruição intracelular dos parasitas (Manna et al., 2015; Sabaté et al., 2014).

$\mathrm{O}$ animal deste trabalho faz o uso de coleira e não recebeu imunoterapia contra a leishmaniose. A vacinação associada ao uso de coleira e inseticidas tópicos são considerados as formas mais efetivas de prevenção e controle da leishmaniose (Werneck et al., 2002). A vacina disponível no mercado apresenta baixa eficácia protetora (Leish-Tec ${ }^{\circledR}-71 \%$ ) (Fernandes et al., 2014; Oliva et al., 2014). Além do mais, não existem evidências científicas de que a eutanásia em cães soropositivos possa reduzir os casos de leishmaniose (Costa \& Vieira, 2001).

\section{Conclusão}

A leishmaniose é uma grave doença que afeta várias espécies, inclusive o homem, na qual o animal pode-se manter assintomático e servir como reservatório para perpetuação dessa afecção. Os métodos para diagnósticos não são totalmente eficazes, sendo um diferencial para diversas outras doenças. $\mathrm{O}$ tratamento tem custo elevado e necessita de monitoração constante pelo veterinário. Podem ainda ocorrer recidivas dos sinais clínicos e muitos tutores acabam por optar pela eutanásia.

\section{Referências bibliográficas}

Abrantes, P. \& Silveira, H. (2009). Alterações climáticas na Europa: efeito nas doenças parasitárias humanas. Revista Portuguesa de Saúde Pública, 27(2):71-86.

Agut, A., Corzo, N., Murciano, J., Laredo, F. G. \& Soler, M. (2003). Clinical and radiographic study of bone and joint lesions in 26 dogs with leishmaniasis. Veterinary Record, 153(21):648-652.

Baneth, G. \& Shaw, S. E. (2002). Chemotherapy of canine leishmaniosis. Veterinary Parasitology, 106(4):315-324.

Benites, A. P., Fernandes, C. E., Brum, K. B. \& Abdo, M. A. G. S. (2011). Presença de formas amastigotas de Leishmania chagasi e perfil leucocitário no aparelho reprodutivo de cães. Pesquisa Veterinária Brasileira, 31(1):72-77.

Blavier, A., Keroack, S., Denerolle, P. H., Goy-Thollot, I., Chabanne, L., Cadore, J. L. \& Bourdoiseau, G. (2001). Atypical forms of canine leishmaniosis. The Veterinary Journal, 162(2):108-120.

Ciaramella, P. \& Corona, M. (2003). Canine leishmaniasis: clinical and diagnostic aspects. Compeindium on Continuing Education for the Practising Veterinarina-North American Edition, 25(5):358-369.

Costa, C. H. N. \& Vieira, J. B. F. (2001). Changes in the control program of visceral leishmaniasis in Brazil. Revista da Sociedade Brasileira de Medicina Tropical, 34(2):223-228.

Cucinotta, G., Iannelli, N., Musicò, M. \& Lombardo, N. (1991). Singolare forma di leishmaniosi: lesioni osteo-articolari bilaterali del carpo e del tarso in un cane. Praxis Veterinaria, 12(3):12-14.

Dantas-Torres, F., Sales, K. G. S., Silva, L. G., Otranto, D. \& Figueredo, L. A. (2017). LeishmaniaFAST15: a rapid, sensitive and low-cost real-time PCR assay for the detection of Leishmania infantum and Leishmania braziliensis kinetoplast DNA in canine blood samples. Molecular and Cellular Probes, 3165-69.

Fernandes, C. B., Junior, J. T. M., Jesus, C., Souza, B. M. P. S., Larangeira, D. F., Fraga, D. B. M., Barrouin-Melo, S. M. (2014). Comparison of two commercial vaccines against visceral leishmaniasis in dogs from endemic areas: $\mathrm{IgG}$, and subclasses, parasitism, and parasite transmission by xenodiagnosis. Vaccine, 32(11):1287-1295.

Ferreira, E. C., Lana, M., Carneiro, M., Reis, A. B., Paes, D. V., Silva, E. S. \& Gontijo, C. M. F. (2007). Comparison of serological assays for the diagnosis of canine visceral leishmaniasis in animals presenting different clinical manifestations. Veterinary Parasitology, 146(3-4):235-241.

Ferrer, L. (1992). Leishmaniasis. In: Kirk, R.W., Bonagura, J.D. Current veterinary therapy: small animal practice. Philadelphia: W.B. Saunders.

Finco, D. R. (1971). Simultaneous determination of phenolsulfonphthalein excretion and endogenous creatinine clearance in the normal dog. Journal of the American Veterinary Medical Association, 159(3):336-340.

Fischer, C., Voss, A. \& Engel, J. (2001). Development status of miltefosine as first oral drug in visceral and cutaneous leishmaniasis. Medical microbiology and immunology, 190(1-2):85-87. 
Gontijo, C. M. F. \& Melo, M. N. (2004). Leishmaniose visceral no Brasil: quadro atual, desfios e perspectivas. Revista Brasileira de Epidemiologia, 7(3):338-349.

Kaneko, J. J., Harvey, J. W. \& Bruss, M. L. (2008). Clinical biochemistry of domestic animals (Vol. 1). New York: Academic press.

Koutinas, A. F. \& Koutinas, C. K. (2014). Pathologic mechanisms underlying the clinical findings in canine leishmaniosis due to Leishmania infantum/chagasi. Veterinary Pathology, 51(2):527-538.

Lanis, A. B., Fonseca, L. A., Roesler, T., Alves, A. \& Lopes, B. (2008). Avaliação laboratorial das doenças renais em pequenos animais. PUBVET, 2(28):1-10.

Madeira, M. F., Uchôa, C. M. A., Leal, C. A., Silva, R. M. M., Duarte, R., Magalhães, C. M. \& Serra, C. M. B. (2003). Leishmania (Viannia) braziliensis in naturally infected dogs. Revista da Sociedade Brasileira de Medicina Tropical, 36(5):551-555.

Mancianti, F., Gramiccia, M., Gradoni, L. \& Pieri, S. (1988). Studies on canine leishmaniasis control. 1. Evolution of infection of different clinical forms of canine leishmaniasis following antimonial treatment. Transactions of the Royal Society of Tropical Medicine and Hygiene, 82(4):566-567.

Manna, L., Corso, R., Galiero, G., Cerrone, A., Muzj, P. \& Gravino, A. E. (2015). Long-term follow-up of dogs with leishmaniosis treated with meglumine antimoniate plus allopurinol versus miltefosine plus allopurinol. Parasites \& Vectors, 81-9.

McConkey, S. E., Lopez, A., Shaw, D. \& Calder, J. (2002). Leishmanial polyarthritis in a dog. The Canadian Veterinary Journal, 43(8):607-609.

Mohapatra, S., Samantaray, J. C., Dash, S. \& Ramakrishan, L. (2014). Lipid derangement as diagnostic and prognostic indicator for visceral leishmaniasis patients. Tropical Parasitology, 4(2):134-135.

Nelson, R. W. \& Couto, C. G. (2015). Medicina interna de pequenos animais. Amsterdan: Elsevier Editora.

Oliva, G., Nieto, J., Manzillo, V. F., Cappiello, S., Fiorentino, E., Di Muccio, T., Carrillo, E. (2014). A randomised, double-blind, controlled efficacy trial of the LiESP/QA-21 vaccine in naïve dogs exposed to two Leishmania infantum transmission seasons. PLoS Neglected Tropical Diseases, 8(10):e3213.

Paz, G. F., Ribeiro, M. F. B., Magalhães, D. F., Sathler, K. P. B., Morais, M. H. F., Fiúza, V. O. P., Dias, E. S. (2010). Association between the prevalence of infestation by Rhipicephalus sanguineus and Ctenocephalides felis felis and the presence of anti-Leishmania antibodies: A case-control study in dogs from a Brazilian endemic area. Preventive Veterinary Medicine, 97(2):131-133.

Pinto, A. J. W., Ribeiro, V. M. \& Tafuri, W. L. (2016). Análise do diagnóstico da leishmaniose visceral canina no Brasil, com ênfase no uso dos métodos sorológicos: teste imunocromatográfico, ELISA e reação de imunofluorescência indireta revisão de literatura. Clinica Veterinária, 21(123):80-86.

Sabaté, D., Llinás, J., Homedes, J., Sust, M. \& Ferrer, L. (2014). A single-centre, open-label, controlled, randomized clinical trial to assess the preventive efficacy of a domperidone-based treatment programme against clinical canine leishmaniasis in a high prevalence area. Preventive Veterinary Medicine, 115(1-2):56-63.

Silva, F. S. (2007). Patologia e patogênese da leishmaniose visceral canina. Revista Tropical-Ciencias Agrarias e Biologicas, 1(1):20-31.

Solano-Gallego, L., Miró, G., Koutinas, A., Cardoso, L., Pennisi, M. G., Ferrer, L., Baneth, G. (2011). LeishVet guidelines for the practical management of canine leishmaniosis. Parasites \& Vectors, 4(86):1-16.

Torres, M., Pastor, J., Roura, X., Tabar, M. D., Espada, Y., Font, A., Planellas, M. (2016). Adverse urinary effects of allopurinol in dogs with leishmaniasis. Journal of Small Animal Practice, 57(6):299-304.

Werneck, G. L., Costa, C. H. N., Walker, A. M., David, J. R., Wand, M. \& Maguire, J. H. (2002). The urban spread of visceral leishmaniasis: clues from spatial analysis. Epidemiology, 13(3):364-367.

Recebido: 10 de março, 2019.

Aprovado: 1 de abril, 2019.

Publicado: 18 de abril, 2019.

Licenciamento: Este artigo é publicado na modalidade Acesso Aberto sob a licença Creative Commons Atribuição 4.0 (CC-BY 4.0), a qual permite uso irrestrito, distribuição, reprodução em qualquer meio, desde que o autor e a fonte sejam devidamente creditados. 